\title{
Clinical and safety evaluation of Brillia patients with attention-deficit/hyperactivity disorder
}

\author{
Timothy Allen ${ }^{*}$, Rashash Dhoalokia ${ }^{2}$, Sylvia Johnson-Park ${ }^{3}$, Giridhar MNV ${ }^{1}$ and Naveed Basha Court ${ }^{1}$ \\ ${ }^{1}$ Global Allied Pharmaceuticals, Center for Excellence in Research and Development, 160 Vista Oak Dr. Longwood, FL 32779, USA \\ ${ }^{2}$ Orange Psychiatric Associates, PA, University of Florida Allied Health, USA \\ ${ }^{3}$ Lake Nona Health, PLLC, University of Florida Allied Health, USA
}

\begin{abstract}
Background: Attention-Deficit/Hyperactivity Disorder (ADHD) is one of the most common neurodevelopmental disorders in children. It is usually first diagnosed in childhood and often lasts into adulthood. Children with ADHD will have trouble paying attention, controlling impulsive behaviors, or be overly active. The hallmarks are hyperactivity, impulsivity, and inattention that are beyond reasonable developmental expectations for a child's age. The science behind Brillia is based on a new way to combine homeopathy and antibody science. The active ingredient of Brillia, Lapine S-100 immune globulin, is produced using antibodies to the brainspecific S-100 protein (S-100B). This protein is an essential regulator of many different intracellular and extracellular brain processes, e.g., various enzyme activities, calcium homeostasis, communication between neurons, etc. Thus, the clinical study of the efficacy of Brillia for Children for the treatment of anxiety disorders, disturbances of behavior, and attention, accompanied by increased excitability, irritability, and hyperactivity, are very important and promising. To determine the efficacy of Brillia for Children for the treatment of anxiety disorders, disturbances of behavior, and attention, accompanied by increased excitability, irritability, and hyperactivity in children, two clinical studies were carried out.
\end{abstract}

Methods: A double-blind, randomized placebo-controlled study of Brillia evaluating clinical efficacy and safety for children with attention deficit hyperactivity disorder was conducted. Prior to inclusion in the study, the patients or their legal guardians were provided with information about the study and signed an informed consent form. Patient information collected during the study is strictly confidential.

\begin{abstract}
Abbreviation: ADD: Attention deficit disorder; ADHD: AttentionDeficit/Hyperactivity Disorder; ANCOVA: Analysis of Covariance; BCM: Behavioral classroom management; CGI: Clinical Global Impression Scale; CNS: Central Nervous System; DBP: Diastolic blood pressure; DSM: Diagnostic and Statistical Manual of Mental Disorders; HR: Heart rate; ICD: International classification of diseases; SBP: Systolic blood pressure; SSSI: Selective serotonin reuptake inhibitors; ADHD: Attention-Deficit/Hyperactivity Disorder.
\end{abstract}

\section{Introduction}

Attention-Deficit/Hyperactivity Disorder (ADHD) is one of the most common neurodevelopmental disorders in children. The hallmarks are hyperactivity, impulsivity, and inattention that are beyond reasonable developmental expectations for a child's age. The diagnosis of ADHD is made frequently in children with behavioral problems or academic underachievement. It is usually first diagnosed in childhood and often lasts into adolescence and adulthood. Children with ADHD typically have trouble paying attention, controlling impulsive behaviors (may act without thinking about what the result will be), or be overly active. Although the use of a standardized approach ensures the reliability of the diagnosis, concerns regarding the validity of the diagnosis often arise. At present, there is no biologic marker that identifies children with ADHD. Furthermore, whether ADHD is a unique disorder or merely one end of the continuum of age-appropriate behavior is not clear.

ADHD incidence rates are 5 to 10 times higher in the United States compared with other countries. There is significant regional variability in the diagnosis and treatment of ADHD across the United States, as well.
For example, $8 \%$ to $10 \%$ of 30,000 children in second to fifth grade were diagnosed with ADHD in 1 Virginia school system, whereas the NIH reports a lower $3 \%$ to $5 \%$ incidence. Underdiagnosis and suboptimal treatment of children with ADHD is also a well-documented public health issue. One study of treatment services for ADHD nationwide found that only $50 \%$ of children with identified ADHD in real-world practice settings receive care that corresponds to guidelines of the American Academy of Child and Adolescent Psychiatry. Barriers to appropriate service provision include a lack of pediatric specialists.

Disorders in behavior and attention are quite common among the pediatric population. The core symptoms include inattention, hyperactivity, and impulsivity. The incidence of Attention-Deficit/ Hyperactivity Disorder (ADHD) children is up to 3-7\%. Approximately $30-80 \%$ of children with ADHD (ADD) have the disorder as adults. Most experts believe that the rate is well above $50 \%$.

The science behind Brillia is based on a new way to combine homeopathy and antibody science. This product has been used for almost ten years in Europe (under different brand names but with the

*Correspondence to: Timothy Allen, Global Allied Pharmaceuticals, Center for Excellence in Research and Development, 160 Vista Oak Dr. Longwood, FL 32779, USA, E-mail: timallenmed69@gmail.com

Key words: attention-deficit/hyperactivity disorder (ADHD), brillia, brain-specific S-100 protein (S-100B), GABA-mimetic and neurotrophic effects

Received: November 01, 2019; Accepted: November 13, 2019; Published: November 16, 2019 
same formulation) and has shown excellent results for children who have been diagnosed with Attention Deficit Hyperactivity Disorder (ADHD), or who are dealing with anxiety and hyperactivity issues. Brillia is found both safe and impactful and is widely accepted as a safe alternative to prescription pharmaceuticals that have harmful side effects. Brillia can help reduce the symptoms of anxiety and hyperactivity, consequently helping improve attention and focus. It also helps in lowering temper tantrums, obsessive behavior, and defiant behavior [1-10].

Drug therapy of anxiety disorders is also a challenge and should be combined with effective psychotherapy. The first-line drugs for the treatment of childhood anxiety disorders are selective serotonin reuptake inhibitors (SSRIs) with broad-spectrum activity but have delayed the onset of action. Benzodiazepines are usually not used as first-line therapy in children and adolescents, as they were shown to cause behavioral disinhibition in younger children and their addictive potential. However, despite the variety of drugs for the treatment of anxiety disorders, their administration is limited by frequent and severe adverse events (Arena, Rozenbaum, 2004; Khodarev, 2002). Brillia is useful in childhood anxiety disorders, unlike other ADHD drugs, which is an additional benefit.

The active ingredient of Brillia is produced using antibodies to the brain-specific S-100 protein (S-100B). This protein is an essential regulator of many different intracellular and extracellular brain processes, e.g., various enzyme activities, calcium homeostasis, communication between neurons, etc. Since almost all mental and neurological diseases, as well as temporal stress-induced conditions, are accompanied by disturbance of the processes as mentioned above, especially communication between neurons, the normalization of these processes is considered to be a prospective way to treat people with such undesirable conditions.

Behavioral Interventions: The effectiveness of behavioral interventions was compared with methylphenidate therapy in the Multimodal Treatment Study of Children With Attention-Deficit/ Hyperactivity Disorder (MTA). This landmark study included 579 children aged 7-10 years with DSM-IV ADHD combined type. There were 3 active treatments (methylphenidate, behavioral therapy, and combination methylphenidate and behavioral treatment), which were compared with community care or "naturalistic" treatment. This controlled multicenter study was continued for 14 months. All active treatments received ongoing monitoring and coordination with parents, teachers, and clinicians. Community care included stimulant therapy in two-thirds of cases, but there was no systematic coordination of care. Parent training was an integral part of behavioral interventions. Parent training included education on ADHD, counseling for parents, training in contingency management techniques, and development of realistic expectations of treatment.

Mechanism of Action: S-100 protein is involved in multiple neurotransmitter networks of the body. Through its effects on these networks, the product helps improve the concentration of attention, reduce hyperactivity, and mitigate anxiety. Brillia for Children exhibits a neuroprotective effect that restricts the area of damage and improves cognitive function (learning achievement and memory). Also, the drug modifies the activity of S-100 protein that integrates synaptic and metabolic processes in the brain. Brain-specific S-100 is expressed in different cell types, including astrocytes and specific neuronal populations. It stimulates cell proliferation and migration and inhibits apoptosis and differentiation in nanomolar concentrations. Extracellular brain-specific S-100 protein regulates synaptic plasticity, although the molecular mechanism of this activity is unknown.
Brillia exerts its therapeutic effects via influencing (normalizing) processes related to the S-100B protein (including different neurotransmitter systems activities). Brillia is an easy melt tablet with no colors or flavor that the child takes throughout the day (between 2-6 pills a day depending on the severity of the symptoms).

Brillia for Children is a new drug based on ultra-low doses of antibodies to endogenous regulators. The molecular target of Brillia for Children is S-100 protein that couples synaptic and metabolic processes in the brain. Brillia for Children has GABA-mimetic and neurotrophic effects, increases the activity of stress-limiting systems, and improves neuronal plasticity. The drug has a broad spectrum of pharmacological action due to the involvement of the central regulatory systems of CNS.

Thus, monotherapy with Brillia for Children neutralized all clinical manifestations of disturbances of behavior and attention (hyperactivity, behavioral disorders, and cognitive deficit), improved school performance, and reduced severity of accompanying asthenic and neurotic disorders in patients.

\section{Treatment and management}

The therapeutic approach to ADHD has been shifting. In some cases, environmental restructuring and behavioral therapy alone has been effective. Developments in behavioral parent training (BPT) and behavioral classroom management (BCM) have also proven useful. Furthermore, behavioral psychotherapy often is successful when used in conjunction with an effective medication regimen. The medications of choice are stimulants, and for adults with ADHD, stimulants represent the best first-line therapeutic option.

\section{Medical care}

Stimulants (methylphenidate, dextroamphetamine): Regarding medication for ADHD, stimulants are the first-line therapy and probably the most effective treatment. All stimulants have similar efficacy but differ by dosing, duration of action, and adverse effect profiles in individual patients. Care should be made to start at the lowest dose and titrate up for clinical efficacy or to intolerance.

Other medications: Atomoxetine (Strattera) has become a secondline and, in some cases, first-line treatment in children and adults with ADHD because of its efficacy and classification as a non-stimulant. However, studies have reported that the overall effect of atomoxetine has not been as extensive as that reported of stimulants. Data suggest that bupropion or venlafaxine may be sufficient. Dosages are similar to those used to treat depression.

Tricyclic antidepressants (imipramine, desipramine, nortriptyline) have been found useful in numerous studies in children with ADHD; however, because of potential adverse effects, they are rarely used for this purpose. Modafinil (Provigil) has recent placebo-controlled data supporting its efficacy in children with ADHD. This medication may currently be used as a third- or fourth-line treatment. Blader et al. evaluated the ability of Divalproex to reduce aggressive behavior in children with ADHD and a disruptive disorder. A higher proportion of improved behavior was observed in the Divalproex group (8 of 14 [57\%]) compared with placebo (2 of 13 [15\%]). A larger trial is needed to study the use of Divalproex further to enhance aggressive behavior in patients with ADHD.

Clonidine and guanfacine have been used with mixed reports of efficacy. Sudden deaths have been reported in children taking clonidine 
with methylphenidate at bedtime. Again, the etiology of these deaths is unclear, and this remains a controversial topic. In September 2010, the FDA approved clonidine extended-release (Kapvay) for ADHD as adjunctive therapy to stimulants or as monotherapy.

\section{Methodology}

\section{Clinical study design}

Study TD1061511-01.18.P: A multicenter, double-blind, placebocontrolled, randomized trial of the Efficacy and safety of Brillia for Children (2 tablets 2 times daily for 12 weeks) in children with Attention Deficit Hyperactivity Disorder, ADHD (Phase IV).

The objective of the study was to assess the clinical efficacy and safety of Brillia for Children in children with ADHD.

\section{Study participants}

Materials and methods: Patients included those aged in between 06-12 years with Diagnosis of ADHD according to DSM-IV with severity Mild to severe (CGI-ADHD-S).

The other inclusion criteria included:

- Clinical signs of ADHD of at least 22 points (ADHD-RS-IV Home Version);

- Absence of thyroid gland pathology;

- Absence of significant impairment of general intelligence;

- Lack of pharmacotherapy for ADHD within 2 weeks prior to the study;

- Informed consent form signed by the child's legal representative.

Scales used for efficacy assessment:

- ADHD Rating Scale-IV Home Version

- Conners scale

- Clinical Global Impression Scale CGI-ADHD-Scale

- The severity of anxiety disorders scales

Randomization and Masking: The study included 100 children, who were randomized into two groups:

1. Treatment group $(\mathrm{n}=50)$ was administered Brillia for Children (2 tablets b.i.d.) for 12 weeks;

2. Control group ( $n=50)$ was administered placebo ( 2 tablets b.i.d.) for 12 weeks.

\section{Discussion of both the Clinical study TD1061511-01.18.P}

Study populations (Brillia for Children, tablets): The study for the Efficacy of Brillia for Children included 198 patients, 98 children were administered Brillia for Children. All 100 patients completed the study of Brillia for Children's efficacy in the treatment of disturbances of behavior and attention.

Brillia for Children experimental groups were compared with placebo groups.

The demographic characteristics of the patients are presented in (Table 1). In all cases, treatment and control groups were comparable by sex, age, the severity of the initial state, comorbidities. The sample size was sufficient for statistical analysis and assessment of clinical efficacy.

Disturbances of behavior and attention: The treatment efficacy of disorders of behavior and attention was analyzed according to standard scales and questionnaires designed to assess the ADHD severity: ADHD-RS-IV Home Version, CGI-ADHD-S, and Conners scale.

Monotherapy with Brillia for Children neutralized all clinical manifestations of disturbances of behavior and attention (hyperactivity, behavioral disorders, cognitive deficit), improved school performance, reduced severity of accompanying asthenic and neurotic disorders in patients.

By the end of the 12-week treatment with Brillia for Children, inattention and hyperactivity/impulsivity decreased by $34.5 \%$ and $42.4 \%$, respectively, according to ADHD-RS- IV Home Version. Monotherapy with Brillia for Children also increased the number of patients with mild ADHD and reduced the number of patients with moderate ADHD. According to CGI-ADHD-S, the total score decreased by $10 \%$ in the treatment group. Conner's scale showed positive dynamics of opposition in a child's behavior, cognitive impairment, hyperactivity, ADHD index. Under all questionnaires and scales, the efficacy of Brillia for Children was superior to placebo.

Thus, Brillia for Children is a highly effective drug and can be recommended for children 5-18 plus years old suffering from disturbances of behavior and attention.

\section{Discussion of Results in Sub-populations (Brillia for Children, tablets)}

Efficacy of Brillia for Children in various age groups: Subpopulations were not identified in clinical study TD1061511-01.18. $\mathrm{P}$ and; therefore, the analysis of the efficacy in sub-populations was not performed.

Table 1. Demographic characteristics of patients (abs (\%))

\begin{tabular}{|c|c|c|c|c|}
\hline \multicolumn{2}{|c|}{ Parameter } & Brillia for Children & Placebo & Total \\
\hline \multicolumn{5}{|c|}{ Clinical Study: TD1061511-01.18.P } \\
\hline \multicolumn{2}{|r|}{ Age, years $(\mathrm{M} \pm \mathrm{m})$} & $9.3 \pm 0.25$ & $9.3 \pm 0.24$ & $6-12$ \\
\hline \multirow{2}{*}{ Sex (abs (\%)) } & M & $38(76.0 \%)$ & $39(78.0 \%)$ & $77(77.0 \%)$ \\
\hline & $\mathrm{F}$ & $12(24.0 \%)$ & $11(22.0 \%)$ & $23(23.0 \%)$ \\
\hline \multicolumn{2}{|c|}{ Weight, $\mathrm{kg}(\mathrm{M} \pm \mathrm{m})$} & $33.2 \pm 1.51$ & $30.9 \pm 0.86$ & - \\
\hline \multicolumn{2}{|c|}{ Height, sm $(\mathrm{M} \pm \mathrm{m})$} & $138.7 \pm 1.71$ & $137.4 \pm 1.38$ & - \\
\hline \multicolumn{2}{|c|}{ Age of diagnosis, years $(\mathrm{M} \pm \mathrm{m})$} & $5.1 \pm 0.28$ & $5.5 \pm 0.24$ & - \\
\hline \multirow{3}{*}{$\begin{array}{l}\text { ADHD type } \\
\text { according to DSM- } \\
\text { IV (abs }(\%))\end{array}$} & Combined & $45(90.0 \%)$ & $50(100 \%)$ & $95(95.0 \%)$ \\
\hline & Predominantly inattentive & $5(10.0 \%)$ & 0 & $5(5.0 \%)$ \\
\hline & $\begin{array}{l}\text { Predominantly hyperactive- } \\
\text { impulsive }\end{array}$ & 0 & 0 & 0 \\
\hline
\end{tabular}


Thus, the analysis of the efficacy of Brillia for Children, depending on the type of ADHD was not possible, due to the insufficient number of enrolled patients with predominantly inattentive and predominantly hyperactive-impulsive types of ADHD.

Discussion of clinical information relevant to dosing recommendations (Brillia for Children, tablets): It is recommended to take 1 or 2 tablets per intake. The drug should be taken 2-3 times daily. The overall duration of treatment is three months; the treatment can be repeated in 1 month, if necessary.

In the study of the efficacy of Brillia for Children for the treatment of anxiety disorders, in children, the following dosage regimens were used:1 tablet t.i.d. The duration of the treatment with Brillia for Children was 12 weeks. The study of the efficacy of Brillia for Children for treatment of disturbances of behavior and attention used following dosage regimen: 2 tablets b.i.d. for 12 weeks.

These schemes of drug administration proved to be effective. However, a particular analysis of the correlation of dosage regimen with efficacy of Brillia for Children was not performed. However, the possibility of flexible-dose titration (2-6 tablets a day), its repeated use during the day, depending on the severity of the disease and current condition of the patient, allows choosing the optimum regimen and achieving positive outcomes in each case.

Thus, the recommended dosage regimen of the drug is efficient and can be used for the treatment of children with anxiety disorders and disturbances of behavior and attention, accompanied by increased excitability, irritability, and hyperactivity.

\section{Persistence of Efficacy and/or Tolerance Effects (Brillia for Children, tablets)}

Note: ${ }^{* * *}-\mathrm{p}<0.001$, significance of differences vs. baseline

A decrease in the efficacy of Brillia for Children with an increase in the duration of therapy was not observed in the study. Thus, longterm use of Brillia for Children does not decrease the efficacy and/or promote tolerance and/or addiction.

Moreover, Brillia for Children gradually reduced inattention and hyperactivity, impulsiveness, cognitive impairment (TD106151101.18.P).

Thus, Brillia for Children led to a gradual decline in both inattention and hyperactivity/impulsiveness in children (Figure 1). Besides, the marked reduction in anxiety severity was reported after two weeks of the drug administration.

Note: ${ }^{*}-p<0.05,{ }^{* *}-p<0.01$, significance of differences vs. baseline

Also, CGI-ADHD-S proved the reduction of ADHD severity (Figure 2). According to the total score dynamics, a 4-week therapy with Brillia for Children significantly reduced ADHD severity.

Note: ${ }^{* *}-p<0.001$, significance of differences vs. baseline

Conner's scale also showed the reduction of the clinical manifestations of ADHD (opposition in child's behavior, cognitive impairment, hyperactivity) (Figure 3). The positive effect of Brillia for Children was registered after four weeks of therapy. By the end of the treatment (12 weeks) total score decreased by $28.8 \%$ [16-21].
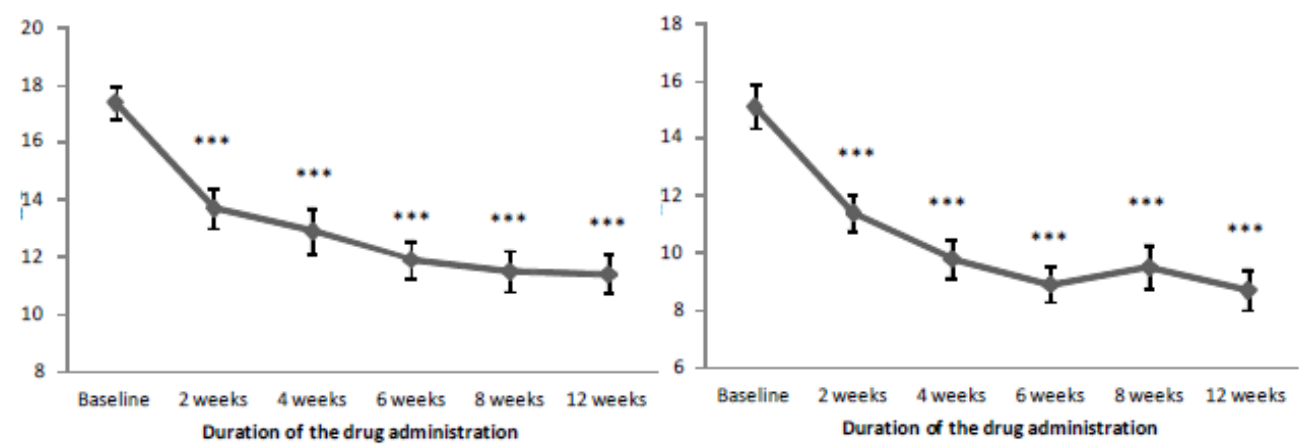

Figure 1. The dynamics of inattention (A) and hyperactivity/impulsiveness (B) according to ADHD-RS-IV Home Version depending on the duration of Brillia for Children administration

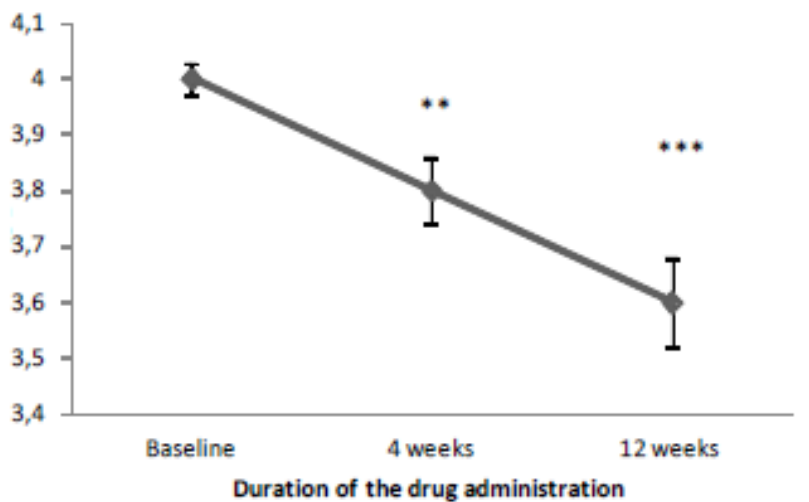

Figure 2. The dynamics of ADHD severity under CGI-ADHD-S depending on the duration of Brillia for Children administration 


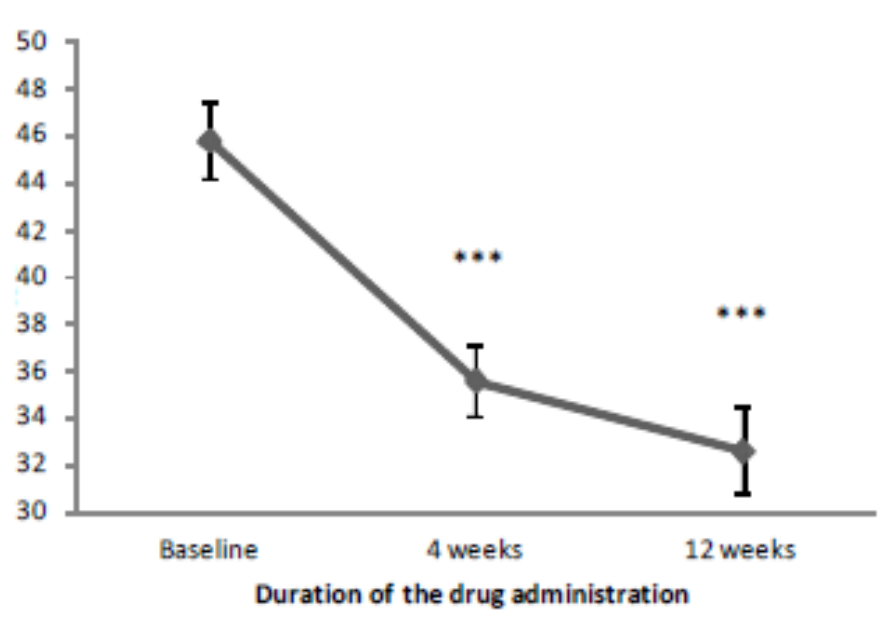

Figure 3. The dynamics of the total score of Connors scale depending on the duration of Brillia for Children administration

\section{Conclusion}

The duration of the treatment with Brillia for Children did not impact the efficacy. Thus, long-term treatment with Brillia for Children does not lead to a decrease in efficacy, the development of tolerance and addiction, but rather to a positive effect, which confirms the need for a long-term course of Brillia for Children.

\section{Results}

\section{Discussion of Clinical Efficacy}

\section{Clinical efficacy for the study TD1061511-01.18.P}

The study included 100 children, who were randomized into two groups:

1. Treatment group $(\mathrm{n}=50)$ was administered Brillia for Children (2 tablets b.i.d.) for 12 weeks;

2. Control group $(n=50)$ was administered placebo ( 2 tablets b.i.d.) for 12 weeks.

\section{Efficacy results on Clinical Study 02:(TD1061511-01.18.P)}

\section{Efficacy criteria:}

1. The proportion of responders;

2. The average reduction of clinical signs of

3. ADHD (ADHD-RS-IV Home Version);

4. The average reduction of $\mathrm{ADHD}$ severity(CGI-ADHD-S);

5. The average reduction of clinical signs of ADHD (Conners scale);

6. The average dynamics of clinical manifestations of ADHD according to the daily assessment of parents.

Note: ${ }^{*}-\mathrm{p}<0.05 ;^{* *}-\mathrm{p}<0.01 ;{ }^{* * *}-\mathrm{p}<0.001$, significance of differences vs. baseline; ${ }^{\circ}-\mathrm{p}<0.05 ;^{\circ \circ}-\mathrm{p}<0.01 ;{ }^{\circ 00}-\mathrm{p}<0.001$, significance of differences vs. baseline

Brillia for Children reduced inattention and hyperactivity/ impulsivity. The total score of the ADHD-RS-IV Home Version decreased by $38.2 \%$ and $18.8 \%$ in the treatment and control group, respectively (Table 2 ).
Note: ${ }^{*}-p<0.05$, the significance of differences vs. placebo

In the treatment group, ADHD severity improved from moderate to mild (Table 3).

Note: $^{* \star}-\mathrm{p}<0.01{ }^{* *}-\mathrm{p}<0.001$, significance of differences vs. baseline

By the end of therapy, Brillia for Children reduced ADHD severity by $10 \%$, placebo - by $5 \%$ (Table 4 ).

Note: ${ }^{*}-\mathrm{p}<0.05 ;^{* *}-\mathrm{p}<0.01 ;{ }^{* * *}-\mathrm{p}<0.001$, significance of differences vs. baseline; ${ }^{\circ}-\mathrm{p}<0.05 ;{ }^{\circ}-\mathrm{p}<0.01 ;{ }^{\circ o \circ}-\mathrm{p}<0.001$, significance of differences vs. placebo

By the end of the study, the total score of the Conners scale decreased by $28.8 \%$ and $13.8 \%$ in the treatment and control group, respectively (Table 5).

Table 2. The results of the ADHD Rating Scale-IV Home Version (points, $\mathrm{M} \pm \mathrm{m}$ )

\begin{tabular}{|c|c|c|}
\hline Treatment phase & Brillia for Children & Placebo \\
\hline \multicolumn{3}{|c|}{ Total score } \\
\hline Baseline & $32.5 \pm 1.14$ & $33.6 \pm 0.96$ \\
\hline 2 weeks & $25.1 \pm 1.03 * * * \circ$ & $28.8 \pm 1.26 * * *$ \\
\hline 4 weeks & $22.7 \pm 1.23 * * * \circ \circ$ & $29.9 \pm 1.06 * * *$ \\
\hline 6 weeks & $20.8 \pm 1.06 * * * \circ \circ$ & $29.0 \pm 1.25 * * *$ \\
\hline 8 weeks & $20.9 \pm 1.30 * * * \circ \circ \circ$ & $27.6 \pm 1.35 * * *$ \\
\hline 12 weeks & $20.1 \pm 1.84 * * * \circ \circ$ & $27.3 \pm 1.48 * * *$ \\
\hline \multicolumn{3}{|c|}{ Inattention } \\
\hline Baseline & $17.4 \pm 0.57$ & $18.4 \pm 0.43$ \\
\hline 2 weeks & $13.7 \pm 0.68 * * * \circ$ & $16.1 \pm 0.66 * * *$ \\
\hline 4 weeks & $12.9 \pm 0.79 * * * \circ \circ \circ$ & $16.4 \pm 0.57 * * *$ \\
\hline 6 weeks & $11.9 \pm 0.64 * * * \circ \circ \circ$ & $16.0 \pm 0.70 * * *$ \\
\hline 8 weeks & $11.5 \pm 0.70 * * * \circ 0 \circ$ & $15.1 \pm 0.76 * * *$ \\
\hline 12 weeks & $11.4 \pm 0.68 * * * \circ \circ$ & $14.9 \pm 0.78 * * *$ \\
\hline \multicolumn{3}{|c|}{ Hyperactivity/impulsiveness } \\
\hline Baseline & $15.1 \pm 0.77$ & $15.2 \pm 0.62$ \\
\hline 2 weeks & $11.4 \pm 0.61 * * *$ & $12.7 \pm 0.74 * * *$ \\
\hline 4 weeks & $9.8 \pm 0.64 * * * \circ \circ \circ$ & $13.5 \pm 0.67 * *$ \\
\hline 6 weeks & $8.9 \pm 0.64 * * * \circ \circ \circ$ & $12.9 \pm 0.73 * *$ \\
\hline 8 weeks & $9.5 \pm 0.76 * * * \circ$ & $12.5 \pm 0.81 * * *$ \\
\hline 12 weeks & $8.7 \pm 0.70 * * * \circ \circ \circ$ & $12.5 \pm 0.82 * * *$ \\
\hline
\end{tabular}

Table 3. ADHD severity according to CGI-ADHD-S (abs (\%))

\begin{tabular}{|c|c|c|}
\hline Treatment phase & Brillia for Children & Placebo \\
\hline & Minor ADHD \\
\hline Baseline & $0(0 \%)$ & $0(0 \%)$ \\
\hline 4 weeks & $0(0 \%)$ & $0(0 \%)$ \\
\hline 12 weeks & $1(2 \%)$ & $0(0 \%)$ \\
\hline \multicolumn{3}{|c|}{ Mild ADHD } \\
\hline Baseline & $2(4.0 \%)$ & $0(0.0 \%)$ \\
\hline 4 weeks & $12(24.0 \%)$ & $6(12.0 \%)$ \\
\hline 12 weeks & $19(38.0 \%)$ & $11(22.0 \%)$ \\
\hline & Moderate ADHD \\
\hline Baseline & $48(96.0 \%)$ & $50(100.0 \%)$ \\
\hline 4 weeks & $38(76.0 \%)$ & $44(88.0 \%)$ \\
\hline 12 weeks & $30(60.0 \%) *$ & $39(78.0 \%)$ \\
\hline
\end{tabular}

Table 4. The total score of CGI-ADHD-S (points, $\mathrm{M} \pm \mathrm{m}$ )

\begin{tabular}{|c|c|c|}
\hline Treatment phase & Brillia for Children & Placebo \\
\hline Baseline & $4.0 \pm 0.03$ & $4.0 \pm 0.00$ \\
\hline 4 weeks & $3.8 \pm 0.06 * *$ & $3.9 \pm 0.05$ \\
\hline 12 weeks & $3.6 \pm 0.08 * * *$ & $3.8 \pm 0.06 * * *$ \\
\hline
\end{tabular}


Table 5. Assessment of ADHD symptoms under the Conners scale (points, $\mathrm{M} \pm \mathrm{m}$ )

\begin{tabular}{|c|c|c|}
\hline Treatment phase & Brillia for Children & \multicolumn{1}{|c|}{ Placebo } \\
\hline & Total score & $50.8 \pm 1.61$ \\
\hline Baseline & $45.8 \pm 1.61$ & $48.0 \pm 1.68 *$ \\
\hline 4 weeks & $35.6 \pm 1.52 * * * \circ \circ$ & $43.8 \pm 2.17 * * *$ \\
\hline 12 weeks & $32.6 \pm 1.84 * * * \circ \circ$ & \\
\hline \multicolumn{3}{|c|}{ Oppositional behavior } \\
\hline Initially & $8.4 \pm 0.46$ & $9.7 \pm 0.51$ \\
\hline 4 weeks & $6.6 \pm 0.44 * * * \circ \circ$ & $9.9 \pm 0.52$ \\
\hline 12 weeks & $6.3 \pm 0.51 * * * \circ$ & $8.4 \pm 0.55$ \\
\hline & Cognitive impairment \\
\hline Baseline & $11.4 \pm 0.50$ & $12.1 \pm 0.50$ \\
\hline 4 weeks & $9.7 \pm 0.56 * * * \circ$ & $12.1 \pm 0.51$ \\
\hline 12 weeks & $8.6 \pm 0.63 * * * \circ \circ$ & $10.9 \pm 0.53 *$ \\
\hline & Hyperactivity \\
\hline Baseline & $9.1 \pm 0.49$ & $11.0 \pm 0.52$ \\
\hline 4 weeks & $6.2 \pm 0.44 * * * \circ \circ$ & $9.2 \pm 0.50 * * *$ \\
\hline 12 weeks & $5.6 \pm 0.39 * * * \circ \circ$ & $9.0 \pm 0.59 * * *$ \\
\hline & ADHD index \\
\hline Baseline & $22.4 \pm 0.78$ & $24.1 \pm 0.71$ \\
\hline 4 weeks & $18.3 \pm 0.81 * * * \circ \circ$ & $23.1 \pm 0.77$ \\
\hline 12 weeks & $16.5 \pm 0.88 * * * \circ \circ$ & $21.4 \pm 0.98 * *$ \\
\hline & &
\end{tabular}

Note: ${ }^{*}-\mathrm{p}<0.05 ;{ }^{* *}-\mathrm{p}<0.01 ;{ }^{* * *}-\mathrm{p}<0.001$, significance of differences vs. baseline; ${ }^{\circ}-\mathrm{p}<0.05 ;^{\circ \circ}-\mathrm{p}<0.01 ;{ }^{\circ 00}-\mathrm{p}<0.001$, significance of differences vs. placebo

The results of the analysis of primary and secondary efficacy criteria were proved with the analysis of daily parents' reports about clinical symptoms of ADHD in children. Brillia for Children significantly decreased total and subscale scores and exceeded the placebo (Table 6).

Conclusions of the efficacy analysis from the clinical study: Brillia for Children had a pronounced therapeutic effect in patients with mild to moderate ADHD. Brillia for Children in a dose of 2 tablets b.i.d. for 12 weeks reduced clinical symptoms of ADHD according to ADHD-RS-IV Home Version and Conners scale, and ADHD severity under CGI-ADHD-S.

Thus, Brillia for Children can be recommended for children with disturbances of behavior and attention.

\section{Safety evaluation:}

\section{Clinical Study (TD1061511-01.18.P) Safety Evaluation}

\section{Safety criteria}

1. Nature of AEs and their relation to study drug;

2. Dynamics of laboratory parameters (complete blood count, urinalysis, and biochemical blood assay).

Safety results: All patients completed the study.

During the study, serious AEs were not reported, and the drug was well-tolerated by all patients.

During the study, 9 cases of AEs were registered, 5 cases were registered in Brillia for Children group, and 4 cases - in the placebo group (Table 7). The safety profile of Brillia for Children was comparable to the placebo. No cases of AEs with certain or probable/ likely relationship with medicinal product intake were registered in the clinical study. The most frequent $\mathrm{AE}$ was an acute upper respiratory infection. It was mild, and investigators and doctors characterized AURI as unlikely related to medicinal product intake. Adverse events were mild and did not require drug discontinuation or administration of other drugs.

Note: HR - heart rate; SBP - systolic blood pressure; DBP - diastolic blood pressure

Analysis of vital parameters revealed no pathological changes during the therapy (Table 8). The differences in visits and between groups were not significant.

Conclusions of the safety analysis from the clinical study: The safety of the study drug was confirmed by the absence of pathological changes in blood and urine tests (Tables 9 and 10). Differences between

Table 6. Dynamics of ADHD symptoms according to parents' assessment (points, M \pm )
\begin{tabular}{|c|c|c|}
\hline Treatment phase & Brillia for Children & Placebo \\
\hline & Total score \\
\hline Baseline & $12.7 \pm 0.90$ & $13.0 \pm 0.97$ \\
\hline 2 weeks & $9.5 \pm 0.70 * * * \circ$ & $12.1 \pm 1.05$ \\
\hline 4 weeks & $9.4 \pm 0.66 * * *$ & $10.9 \pm 0.72 *$ \\
\hline 8 weeks & $7.3 \pm 0.85 * * * \circ \circ$ & $12.5 \pm 0.89$ \\
\hline 12 weeks & $6.8 \pm 0.74 * * * \circ$ & $10.0 \pm 0.99 *$ \\
\hline & Morning subscale score \\
\hline Baseline & $3.1 \pm 0.35$ & $3.0 \pm 0.38$ \\
\hline 2 weeks & $1.8 \pm 0.24 * * * \circ$ & $2.7 \pm 0.31$ \\
\hline 4 weeks & $1.6 \pm 0.24 * * *$ & $2.4 \pm 0.33$ \\
\hline 8 weeks & $1.4 \pm 0.24 * * * \circ \circ$ & $3.0 \pm 0.31$ \\
\hline 12 weeks & $1.2 \pm 0.20 * * * \circ$ & $2.1 \pm 0.23$ \\
\hline & Night subscale score \\
\hline Baseline & $9.5 \pm 0.66$ & $10.0 \pm 0.72$ \\
\hline 2 weeks & $7.7 \pm 0.56 * *$ & $9.6 \pm 0.79$ \\
\hline 4 weeks & $7.8 \pm 0.54 *$ & $8.7 \pm 0.52 *$ \\
\hline 8 weeks & $5.9 \pm 0.64 * * * \circ \circ$ & $9.4 \pm 0.65$ \\
\hline 12 weeks & $5.5 \pm 0.63 * * * \circ$ & $7.8 \pm 0.80 * *$ \\
\hline & &
\end{tabular}

Table 7: AEs in placebo and Brillia for Children groups

\begin{tabular}{|c|c|c|c|}
\hline Adverse event & Specification & Brillia $(\mathrm{n}=50)$ & Placebo $(n=50)$ \\
\hline \multirow{3}{*}{ AURI } & Frequency, n (\%) & $2(4 \%)$ & $2(4 \%)$ \\
\hline & Severity & Mild / Moderate & Mild / Moderate \\
\hline & $\begin{array}{l}\text { Relationship with } \\
\text { treatment }\end{array}$ & Unlikely & Unlikely \\
\hline \multirow{3}{*}{ Acute bronchitis } & Frequency, n (\%) & \multirow[b]{3}{*}{-} & $1(2 \%)$ \\
\hline & Severity & & Mild \\
\hline & $\begin{array}{l}\text { Relationship with } \\
\text { treatment }\end{array}$ & & Unlikely \\
\hline \multirow{3}{*}{ Sleepwalking } & Frequency, n (\%) & \multirow[b]{3}{*}{ - } & $1(2 \%)$ \\
\hline & Severity & & Mild \\
\hline & $\begin{array}{l}\text { Relationship with } \\
\text { treatment }\end{array}$ & & Unlikely \\
\hline \multirow{3}{*}{ Headaches } & Frequency, n (\%) & $1(2 \%)$ & \multirow[b]{3}{*}{ - } \\
\hline & Severity & Mild & \\
\hline & $\begin{array}{l}\text { Relationship with } \\
\text { treatment }\end{array}$ & Unlikely & \\
\hline \multirow{3}{*}{$\begin{array}{l}\text { Impaired glucose } \\
\text { tolerance }\end{array}$} & Frequency, n (\%) & $1(2 \%)$ & \multirow[b]{3}{*}{-} \\
\hline & Severity & Mild & \\
\hline & $\begin{array}{l}\text { Relationship with } \\
\text { treatment }\end{array}$ & Unlikely & \\
\hline \multirow{3}{*}{$\begin{array}{c}\text { Hay fever } \\
\text { (exacerbation) }\end{array}$} & Frequency, n (\%) & $1(2 \%)$ & \multirow[b]{3}{*}{-} \\
\hline & Severity & Mild & \\
\hline & $\begin{array}{c}\text { Relationship with } \\
\text { treatment }\end{array}$ & Unlikely & \\
\hline \multicolumn{2}{|c|}{ Total: } & 14 & 12 \\
\hline
\end{tabular}


Table 8. Dynamics of vital parameters $(\mathrm{M} \pm \mathrm{m})$

\begin{tabular}{|c|c|c|c|c|c|c|}
\hline \multirow{2}{*}{ Index } & \multicolumn{3}{|c|}{ Brillia for Children } & \multicolumn{3}{|c|}{ Placebo } \\
\hline & Baseline & 4 weeks & 12 weeks & Baseline & 4 weeks & 12 weeks \\
\hline HR, bpm & $84.4 \pm 1.64$ & $84.0 \pm 1.17$ & $81.0 \pm 0.74$ & $82.7 \pm 1.04$ & $84.4 \pm 1.64$ & $84.0 \pm 1.17$ \\
\hline SBP, mmHg & $100.4 \pm 1.57$ & $98.3 \pm 1.26$ & $98.1 \pm 1.41$ & $95.7 \pm 1.45$ & $100.4 \pm 1.57$ & $98.3 \pm 1.26$ \\
\hline DBP, mmHg & $68.7 \pm 1.21$ & $66.9 \pm 0.73$ & $65.5 \pm 0.81$ & $63.9 \pm 0.76$ & $68.7 \pm 1.21$ & $66.9 \pm 0.73$ \\
\hline Body temperature, ${ }^{\circ} \mathrm{C}$ & $36.6 \pm 0.02$ & $36.5 \pm 0.02$ & $36.6 \pm 0.02$ & $36.5 \pm 0.02$ & $36.6 \pm 0.02$ & $36.5 \pm 0.02$ \\
\hline
\end{tabular}

Table 9. Dynamics of complete blood count $(\mathrm{M} \pm \mathrm{m})$

\begin{tabular}{|c|c|c|c|c|c|}
\hline \multirow{2}{*}{ Parameter } & \multirow{2}{*}{ Standard } & \multicolumn{2}{|c|}{ Brillia for Children } & \multicolumn{2}{|c|}{ Placebo } \\
\hline & & Baseline & 12 weeks & Baseline & 12 weeks \\
\hline $\operatorname{ESR}(\mathrm{mm} / \mathrm{h})$ & $0.0-15.0$ & $6.4 \pm 0.97$ & $8.5 \pm 1.05$ & $6.6 \pm 1.11$ & $6.9 \pm 0.71$ \\
\hline Leucocytes $\left(10^{3} / \mu 1\right)$ & $4.5-13.5$ & $6.1 \pm 0.19$ & $7.3 \pm 0.34$ & $6.2 \pm 0.22$ & $6.3 \pm 0.24$ \\
\hline Hemoglobin $(\mathrm{g} / \mathrm{l})$ & $115.0-145.0$ & $129.8 \pm 1.17$ & $132.0 \pm 1.23$ & $131.4 \pm 0.89$ & $129.4 \pm 1.06$ \\
\hline Erythrocytes $\left(10^{6} / \mu 1\right)$ & $3.8-4.9$ & $4.6 \pm 0.03$ & $4.7 \pm 0,04$ & $4.7 \pm 0 ., 04$ & $4.7 \pm 0.04$ \\
\hline Hematocrit $(\%)$ & $33.0-41.0$ & $37.7 \pm 0.25$ & $38.7 \pm 0.29$ & $37.5 \pm 0.58$ & $37.6 \pm 0.36$ \\
\hline Thrombocytes $\left(10^{3} / \mu 1\right)$ & $150.0-400.0$ & $301.4 \pm 7.86$ & $303.9 \pm 7.02$ & $313.9 \pm 8.18$ & $305.6 \pm 8.07$ \\
\hline Neutrophils (\%) & $42.0-66.0$ & $41.6 \pm 1.63$ & $49.6 \pm 1.72$ & $44.9 \pm 1.17$ & $43.4 \pm 1.44$ \\
\hline Basophils (\%) & $<1.0$ & $0.5 \pm 0.09$ & $0.3 \pm 0.05$ & $0.3 \pm 0.05$ & $0.5 \pm 0.08$ \\
\hline Eosinophiles (\%) & $1.0-5.0$ & $5.1 \pm 0.70$ & $4.2 \pm 0.49$ & $4.4 \pm 0.37$ & $4.5 \pm 0.46$ \\
\hline Monocytes (\%) & $3.0-9.0$ & $10.7 \pm 0.96$ & $9.6 \pm 0.44$ & $10.6 \pm 1.22$ & $9.4 \pm 0.33$ \\
\hline Lymphocytes (\%) & $30.0-50.0$ & $42.1 \pm 1.42$ & $36.1 \pm 1.82$ & $41.0 \pm 1.17$ & $42.1 \pm 1.34$ \\
\hline
\end{tabular}

Table 10. Dynamics of urinalysis

\begin{tabular}{|c|c|c|c|c|}
\hline \multirow{2}{*}{ Parameter } & \multirow{2}{*}{ Standard } & \multicolumn{2}{|c|}{ Brillia for Children } & \multicolumn{2}{|c|}{ Placebo } \\
\cline { 2 - 4 } & & Baseline & 12 weeks & Baseline \\
\hline Specific gravity $(\mathrm{M} \pm \mathrm{m})$ & $1012.0-1025.0$ & $1024.2 \pm 1.02$ & $1021.7 \pm 1.35$ & $1022.6 \pm 1.07$ \\
\hline Protein, g/l (abs (\%)) & $\leq 0.140$ & $37(74.0 \%)$ & $39(78.0 \%)$ & $40(80.0 \%)$ \\
\hline Glucose (abs (\%)) & absent & $50(100.0 \%)$ & $50(100.0 \%)$ & $40(80.0 \%)$ \\
\hline
\end{tabular}

laboratory tests at visits and in groups were not significant and were in the physiological range. Thus, Brillia for Children (4 tablets a day) for 12 weeks is safe for children with ADHD.

\section{Discussion and conclusion}

The study showed that Brillia for children had a pronounced therapeutic effect in patients with mild to moderate ADHD. Brillia for Children reduced clinical symptoms of ADHD according to ADHDRS-IV home version and conners scale and ADHD severity under CGIADHD-S. Thus, Brillia is recommended for children with disturbances of behavior and attention.

Brillia proved that the monotherapy with Brillia for Children neutralized all clinical manifestations of disturbances of behavior and attention (hyperactivity, behavioral disorders, and cognitive deficit), improved school performance, and reduced severity of accompanying asthenic, and neurotic disorders in patients.

No significant changes were noticed in the clinical and laboratory parameters and were found to be within the physiological deviations by the nature of the influence on clinical and laboratory indices. The use of Brillia in children showed non-inferiority to Placebo. The treatment with Brilia also proved the high degree of safety of the drug. Treatment with Brillia showed a pronounced therapeutic effect in patients with mild to moderate ADHD.

Childhood anxiety disorders are on the rise in recent days with the influence of social media and highly competitive society. The conventional therapies such as SSRIs and SNRIs are playing an essential role in the management of these disorders. However dose titration, treatment emergent adverse events such as obesity, delayed onset of action and more protracted course of therapy are the drawbacks of these therapies. Brillia can be useful in overcoming these challenges as it is a targeted therapy. Brillia can be tried on larger sample size as monotherapy and combination therapies with non-pharmacological managements such as biofeedback, muscle relaxation techniques, etc.

\section{References}

1. American Psychiatric Association (2000) Diagnostic and Statistical Manual of Mental Disorders.

2. Arena J, Rozenbaum J (2004) Pharmacotherapy of mental disorders: Tr. from English. M. Binom, 416.

3. (2011) Clinical Practice Guideline for the Diagnosis, Evaluation, and Treatment of Attention- Deficit/Hyperactivity Disorder in Children and Adolescents, American Academy of Pediatrics.

4. Expert OpinPharmacotherIP, Salazar JAB (2008) Pharmacotherapy of adult attention deficit/hyperactivity disorder: A review of evidence-based practices and future directions. SB - Expert OpinPharmacother 9:1299-1310.

5. Blader JC, Schooler NR, Jensen PS, Pliszka SR, Kafantaris V (2009) Adjunctive divalproex versus placebo for children with ADHD and aggression refractory to stimulant monotherapy. Am J Psychiatry 166: 1392-1401.

6. Commission directive 2003/63/EC of 25 June 2003 amending Directive 2001/83/EC of the European parliament and the council on the community code relating to medicinal products for human use.

7. Donato R, Cannon BR, Sorci G, Riuzzi F, Hsu K, et al. (2013) Functions of S100 proteins. Curr Mol Med 13: 24-57. [Crossref]

8. Edwards SL, Rapee RM, Kennedy SJ, Spence SH (2010) The assessment of anxiety symptoms in preschool-aged children: the revised Preschool Anxiety Scale. J Clin Child Adolesc Psychol 39: 400-409.

9. Constitution of the World Health Organization. Geneva, Official records of WHO, No.2:100. Directive 2001/83/EC of the European Parliament and the Council of 6 November 2001 on the Community code relating to medicinal products for human use.

10. Guidelinesforexperimental(preclinical)studiesofnewpharmacologicalagents (2005) Medicine 832. 
11. Keeton CP, Kolos AC, Walkup JT (2009) Pediatric generalized anxiety disorder: epidemiology, diagnosis, and management. Paediatr Drugs 11: 171-183. [Crossref]

12. Khodarev SV (2002) Approaches to the diagnosis and correction of anxiety disorders in children / Khodarev SV CurrPediat Iss 1: 92-94.

13. Liberman LC, Lipp OV, Spence SH, March S (2006) Evidence for retarded extinction of aversive learning in anxious children. Behav Res Ther 44: 1491-1502.

14. Mash E, Wolf D (2003) Children's psychopathology: Violations of the child's mind: Trans. from English. St. Petersburg: Prime-Evroznak. 384.

15. Mosholder AD, Gelperin K, Hammad TA, Phelan K, Johann-Liang R (2009) Hallucinations, and other psychotic symptoms associated with the use of attentiondeficit/hyperactivity disorder drugs in children. Pediatrics 123: 611-616.

16. Sawyer MG, Pfeiffer S, Spence SH (2009) Life events, coping, and depressive symptoms among young adolescents: a one-year prospective study. $J$ Affect Disord 117: $48-54$.
17. Shear MK, Bjelland I, Beesdo K, Gloster AT, Wittchen HU (2007) Supplementary dimensional assessment in anxiety disorders. Int J Methods Psychiatr Res 16: 5264 .

18. Shmakova OP (2004) School adaptation of children and adolescents with psychiatric disorders: 24

19. Swanson JM, Elliott GR, Greenhill LL, Wigal T, Arnold LE, et al. (2007) Effects of stimulant medication on growth rates across 3 years in the MTA follow-up. J Am Acad Child Adolesc Psychiatry 46: 1015-1027. [Crossref]

20. WenarCh, Kering P (2004) Psychopathology of childhood and adolescence. Developmental psychopathology: Tr. from English. St. Petersburg.: Prime-Evroznak, 384.

21. Zavadenko NN (2006) Attention deficit hyperactivity disorder in children: diagnosis andtreatment. Russian medical J 14: 51-56.

Copyright: (2019 Allen T. This is an open-access article distributed under the terms of the Creative Commons Attribution License, which permits unrestricted use, distribution, and reproduction in any medium, provided the original author and source are credited. 